\title{
Powers under umbral composition and degeneration for Sheffer sequences
}

\author{
Dae San Kim ${ }^{1 \dagger}$, Taekyun Kim ${ }^{2,3{ }^{*}}$, Hyuck In Kwon ${ }^{3 \dagger}$ and Toufik Mansour ${ }^{4 \dagger}$
}

"Correspondence: tkkim@kw.ac.kr

${ }^{2}$ Department of Mathematics,

College of Science, Tianjin

Polytechnic University, Tianjin,

300387, China

${ }^{3}$ Department of Mathematics,

Kwangwoon University, Seoul,

139-701, South Korea

†Equal contributors

Full list of author information is

available at the end of the article

\begin{abstract}
In this paper we study the powers under umbral composition and degeneration for Sheffer sequences, where we presented several applications related to Bernoulli polynomials, Frobenius-Euler polynomials, falling factorial polynomials and Bell polynomials and their degeneration cases.
\end{abstract}

MSC: Primary 05A19; 05A40; secondary 11B83

Keywords: umbral composition; degeneration; Sheffer sequence; umbral calculus; Bernoulli polynomials; Korobov polynomials; falling factorial polynomials; Bell polynomials

\section{Introduction}

The aim of this paper is to use umbral calculus and to study powers under umbral composition and degeneration for Sheffer sequences. Umbral calculus (see $[1,2])$ has been used in numerous problems of applied mathematics, theoretical physics, approximation theory, and several diverse areas of mathematics. In this paper, umbral calculus is considered for some special Sheffer polynomials (to be defined in next section) such as Bell polynomials, Bernoulli polynomials, Frobenius-Euler polynomials, Korobov polynomials, degenerate Bernoulli polynomials, and falling factorial polynomials.

The order $O(f(t))$ of the non-zero power series $f(t)$ is the smallest integer $k$ for which the coefficient of $t^{k}$ does not vanish. A series $g(t)$ with $O(g(t))=0$ is called an invertible series and a series $f(t)$ with $O(f(t))=1$ is called a delta series.

Let $g(t)$ be an invertible series and let $f(t)$ be a delta series. Then there exists a unique sequence $s_{n}(x)$ of polynomials such that $\left\langle g(t) f(t)^{k} \mid s_{n}(x)\right\rangle=n ! \delta_{n, k}$, for $n, k \geq 0$ (see [2]). The sequence $s_{n}(x)$ is called the Sheffer sequence for the Sheffer pair $(g(t), f(t))$, which is indicated by $s_{n}(x) \sim(g(t), f(t))$. It is well known that $s_{n}(x) \sim(g(t), f(t))$ if and only if

$$
\frac{1}{g(\bar{f}(t))} e^{x \bar{f}(t)}=\sum_{n \geq 0} s_{n}(x) \frac{t^{n}}{n !}
$$

where $\bar{f}(t)$ is the compositional inverse of $f(t)$ determined by $f(\bar{f}(t))=\bar{f}(f(t))=t$.

For each nonnegative integer $m$, the $m$ th power of an invertible series $g(t)$ will be indicated by $(g(t))^{m}$, while the compositional powers of a delta series $f(t)$ will be denoted by $f^{m}(t)=f \circ f \circ \cdots \circ f(t)$. For $p_{n}(x)$ and $q_{n}(x)=\sum_{k=0}^{n} q_{n, k} t^{k}$, the umbral composition of $q_{n}(x)$

\section{Springer}


with $p_{n}(x)$, denoted by $q_{n} \circ p_{n}(x)$, is defined by $q_{n} \circ p_{n}(x)=\sum_{k=0}^{n} q_{n, k} p_{k}(x)$. The main goal of this paper is to study the powers under umbral composition and degeneration for Sheffer sequences. Moreover, we present several applications related to Bernoulli polynomials, Frobenius-Euler polynomials, falling factorial polynomials and Bell polynomials and their degeneration cases (for definitions, see below). For instance, see Corollaries 3.2, 3.4, 3.6, and 3.8.

\section{Preliminaries: powers under umbral composition and degeneration for Sheffer sequences}

We start by stating the following theorem, which is given as Theorem 3.5.5 in Roman's book [2].

Theorem 2.1 The set of Sheffer sequences is a group under operation of umbral composition. If $s_{n}(x) \sim(g(t), f(t))$ and $r_{n}(x) \sim(h(t), \ell(t))$, then $r_{n}(x) \circ s_{n}(x) \sim(g(t) h(f(t)), \ell(f(t)))$. The identity under umbral composition is $x^{n} \sim(1, t)$, and the inverse of the sequence $s_{n}(x) \sim(g(t), f(t))$ is the Sheffer sequence for $\left(g(\bar{f}(t))^{-1}, \bar{f}(t)\right)$.

As a corollary, we see that, if $s_{n}(x) \sim(g(t), f(t))$ and $r_{n}(x) \sim(1, \ell(t))$, then the generating function for $r_{n} \circ s_{n}(x)$ is obtained from that for $s_{n}(x)$ by substituting $\bar{\ell}(t)$ for $t$. As $r_{n} \circ s_{n}(x) \sim$ $(g(t), \ell(f(t)))$, and the compositional inverse of $\ell(f(t))$ is $\bar{f}(\bar{\ell}(t))$,

$$
g(\bar{f}(\bar{\ell}(t)))^{-1} e^{x \bar{f}(\bar{\ell}(t))}=\sum_{n \geq 0} r_{n} \circ s_{n}(x) \frac{t^{n}}{n !}
$$

From the definition of umbral composition, we see that the $m$ th power under umbral composition of $r_{n}(x) \sim(h(t), \ell(t))$ is given by

$$
r_{n}^{(m)}(x) \sim\left(\prod_{i=1}^{m-1} h\left(\ell^{i}(t)\right), \ell^{m}(t)\right)
$$

for $m \in \mathbb{Z}_{>0}$. In particular, for the Appel sequence $r_{n}(x) \sim(h(t), t)$, we have $r_{n}^{(m)}(x) \sim$ $\left((h(t))^{m}, t\right)$; for the associated sequence $r_{n}(x) \sim(1, \ell(t))$, we have $r_{n}^{(m)}(x) \sim\left(1, \ell^{m}(t)\right)$.

For $n \geq 0$, we write $r_{n}(x)=\sum_{k=0}^{n} r_{n, k} x^{k}=\sum_{k \geq 0} r_{n, k} x^{k}$, where we agree that $r_{i, j}=0$ for all $i<j$. In general, we write

$$
r_{n}^{(m)}(x)=\sum_{k=0}^{n} r_{n, k}^{(m)} x^{k}=\sum_{k \geq 0} r_{n, k}^{(m)} x^{k},
$$

for all $m \in \mathbb{Z}_{>0}$. Then we see that

$$
\begin{aligned}
r_{n, k}^{(m)} & =\sum_{\ell_{1}, \ldots, \ell_{m-1}=0}^{n} r_{n, \ell_{1}} r_{\ell_{1}, \ell_{2}} \cdots r_{\ell_{m-1}, k}, \quad m \geq 2, \\
r_{n, k}^{(1)} & =r_{n, k}
\end{aligned}
$$


(from now on, the sum in (3) is understood as $r_{n, k}$ for $m=1$ ). From (2), the generating function for $r_{n}^{(m)}(x)$ is given by

$$
\sum_{n \geq 0} r_{n}^{(m)}(x) \frac{t^{n}}{n !}=\frac{1}{\prod_{i=0}^{m-1} h\left(\ell^{i}\left(\bar{\ell}^{m}(t)\right)\right)} e^{x^{\overline{\ell^{m}}(t)}}=\frac{1}{\prod_{i=0}^{m-1} h\left(\bar{\ell}^{m-i}(t)\right)} e^{x \bar{\ell}^{m}(t)}
$$

Let $R=R_{h(t), \ell(t)}$ be the lower triangular matrix of infinite size where rows and columns are indexed by nonnegative integers and the $n$th row consists of the coefficients of $r_{n}(x)$, namely $r_{n, 0}, \ldots, r_{n, n}, 0,0, \ldots$ Then, as was noted in [3], from (3) we see that $r_{n, k}^{(m)}$ is the $(n, k)$ entry of $R^{m}$.

Let $r_{n}(x)=\sum_{k=0}^{n} r_{n, k} x^{k} \sim(h(t), t)$. Thus, by (2), we have

$$
r_{n}^{(m)}(x)=\sum_{k=0}^{n} r_{n, k}^{(m)} x^{k} \sim\left((h(t))^{m}, t\right)
$$

Observe here that the notation $r_{n}^{(m)}(x)$ for the $m$ th power of $r_{n}(x)$ under umbral composition agrees with that for $m$ th order polynomial of $r_{n}(x)$.

Now let us give two examples. At first, let $\alpha>0$. Let us first consider the Bernoulli polynomials $B_{n}^{(\alpha)}(x)$ of order $\alpha$ (see [4]). So, if

$$
r_{n}(x)=B_{n}^{(\alpha)}(x)=\sum_{k=0}^{n}\left(\begin{array}{l}
n \\
k
\end{array}\right) B_{n-k}^{(\alpha)} x^{k} \sim\left(\left(\frac{e^{t}-1}{t}\right)^{\alpha}, t\right),
$$

then

$$
r_{n}^{(m)}(x)=B_{n}^{(\alpha m)}(x)=\sum_{k=0}^{n}\left(\begin{array}{l}
n \\
k
\end{array}\right) B_{n-k}^{(\alpha m)} x^{k} \sim\left(\left(\frac{e^{t}-1}{t}\right)^{\alpha m}, t\right) .
$$

Thus, from (3), we get the following result.

Theorem 2.2 For all $n \geq k \geq 0$ and $\alpha, m \in \mathbb{Z}_{>0}$,

$$
\left(\begin{array}{l}
n \\
k
\end{array}\right) B_{n-k}^{(\alpha m)}=\sum_{\ell_{1}, \ldots, \ell_{m-1}=0}^{n}\left(\begin{array}{l}
n \\
\ell_{1}
\end{array}\right)\left(\begin{array}{l}
\ell_{1} \\
\ell_{2}
\end{array}\right) \cdots\left(\begin{array}{c}
\ell_{m-1} \\
k
\end{array}\right) B_{n-\ell_{1}}^{(\alpha)} B_{\ell_{1}-\ell_{2}}^{(\alpha)} \cdots B_{\ell_{m-1}-k}^{(\alpha)} .
$$

As a second example, let us take the Frobenius-Euler polynomials $H_{n}^{(\alpha)}(x \mid \lambda)$ of order $\alpha$, $1 \neq \lambda \in \mathbb{C}$ (see [5-7]). So, if

$$
r_{n}(x)=H_{n}^{(\alpha)}(x \mid \lambda)=\sum_{k=0}^{n}\left(\begin{array}{l}
n \\
k
\end{array}\right) H_{n, k}^{(\alpha)}(\lambda) x^{k} \sim\left(\left(\frac{e^{t}-\lambda}{1-\lambda}\right)^{\alpha}, t\right),
$$

then

$$
r_{n}^{(m)}(x)=H_{n}^{(\alpha m)}(x \mid \lambda)=\sum_{k=0}^{n}\left(\begin{array}{l}
n \\
k
\end{array}\right) H_{n, k}^{(\alpha m)}(\lambda) x^{k} \sim\left(\left(\frac{e^{t}-\lambda}{1-\lambda}\right)^{\alpha m}, t\right) .
$$

Thus, again from (3), we obtain the following result. 
Theorem 2.3 For all $n \geq k \geq 0$ and $\alpha, m \in \mathbb{Z}_{>0}$,

$$
\left(\begin{array}{l}
n \\
k
\end{array}\right) H_{n-k}^{(\alpha m)}=\sum_{\ell_{1}, \ldots, \ell_{m-1}=0}^{n}\left(\begin{array}{l}
n \\
\ell_{1}
\end{array}\right)\left(\begin{array}{l}
\ell_{1} \\
\ell_{2}
\end{array}\right) \cdots\left(\begin{array}{c}
\ell_{m-1} \\
k
\end{array}\right) H_{n-\ell_{1}}^{(\alpha)}(\lambda) H_{\ell_{1}-\ell_{2}}^{(\alpha)}(\lambda) \cdots H_{\ell_{m-1}-k}^{(\alpha)}(\lambda) .
$$

\section{Powers under umbral composition applied to associated sequences}

In this section, we study the powers under umbral composition applied to associated sequences. Throughout this section, let $r_{n}(x)=\sum_{k=0}^{n} r_{n, k} x^{k} \sim(1, f(t))$ and $r_{n}^{(m)}(x)=$ $\sum_{k=0}^{n} r_{n, k}^{(m)} x^{k} \sim\left(1, f^{m}(t)\right)$.

\subsection{Generalized falling factorial polynomials}

As a first interesting case, let us consider the generalized falling factorial polynomials $(x \mid \lambda)_{n}=x(x-\lambda) \cdots(x-(n-1) \lambda)$, for $n \geq 1$, and $(x \mid \lambda)_{0}=1$ (see [4]). So

$$
r_{n}(x)=(x \mid \lambda)_{n}=\sum_{k=0}^{n} \lambda^{n-k} S_{1}(n, k) x^{k} \sim(1, f(t))
$$

with $f(t)=\frac{e^{\lambda t}-1}{\lambda}$. Then, by (3), we obtain

$$
\begin{aligned}
r_{n, k}^{(m)} & =\sum_{\ell_{1}, \ldots, \ell_{m-1}=0}^{n} \lambda^{n-\ell_{1}} S_{1}\left(n, \ell_{1}\right) \lambda^{\ell_{1}-\ell_{2}} S_{1}\left(\ell_{1}, \ell_{2}\right) \cdots \lambda^{\ell_{m-1}-k} S_{1}\left(\ell_{m-1}, k\right) \\
& =\lambda^{n-k} \sum_{\ell_{1}, \ldots, \ell_{m-1}=0}^{n} S_{1}\left(n, \ell_{1}\right) S_{1}\left(\ell_{1}, \ell_{2}\right) \cdots S_{1}\left(\ell_{m-1}, k\right) .
\end{aligned}
$$

To proceed, we recall the transfer formula (see [2]): for $p_{n}(x) \sim(1, f(t))$ and $q_{n}(x) \sim(1, \ell(t))$, we have $q_{n}(x)=x(f(t) / \ell(t))^{n} x^{-1} p_{n}(x)$, for all $n \geq 1$. As $x^{n} \sim(1, t)$, we have, for $n \geq 1$,

$$
\begin{aligned}
r_{n}(x) & =x\left(\frac{\lambda t}{e^{\lambda t}-1}\right)^{n} x^{-1} x^{n}=x \sum_{k \geq 0} \beta_{k}^{(n)} \frac{\lambda^{k}}{k !} t^{k} x^{n-1} \\
& =x \sum_{k=0}^{n-1}\left(\begin{array}{c}
n-1 \\
k
\end{array}\right) \lambda^{k} \beta_{k}^{(n)} x^{n-1-k}=x \sum_{k=0}^{n-1}\left(\begin{array}{c}
n-1 \\
k
\end{array}\right) \lambda^{n-1-k} \beta_{n-1-k}^{(n)} x^{k} \\
& =\sum_{k=1}^{n}\left(\begin{array}{c}
n-1 \\
k-1
\end{array}\right) \lambda^{n-k} \beta_{n-k}^{(n)} x^{k} .
\end{aligned}
$$

Thus, for $n \geq 1,0 \leq m \leq n-1$, we observe that

$$
(f(t))^{m} x^{-1} r_{n}(x)=(f(t))^{m}(t / f(t))^{n} x^{n-1}=(t / f(t))^{n-m} t^{m} x^{n-1}
$$

Therefore,

$$
(f(t))^{m} x^{-1} r_{n}(x)=\sum_{\ell=0}^{n-1-m} \frac{(n-1)_{\ell+m}}{\ell !} \lambda^{\ell} B_{\ell}^{(n-m)} x^{n-1-\ell-m}
$$


Now, we get, for $n \geq 1, r_{n}^{(2)}(x)=x\left(f(t) / f^{2}(t)\right)^{n} x^{-1} r_{n}(x)$, which gives

$$
\begin{aligned}
& r_{n}^{(2)}(x)=x \sum_{k_{2}=0}^{n-1} B_{k_{2}}^{(n)} \frac{\lambda^{k_{2}}}{k_{2} !}(f(t))^{k_{2}} x^{-1} r_{n}(x) \\
& =\sum_{k_{2}=0}^{n-1} \sum_{k_{1}=0}^{n-1-k_{2}}\left(\begin{array}{c}
n-1 \\
k_{1}, k_{2}, n-1-k_{1}-k_{2}
\end{array}\right) \lambda^{k_{1}+k_{2}} B_{k_{2}}^{(n)} B_{k_{1}}^{\left(n-k_{2}\right)} x^{n-k_{1}-k_{2}} \\
& =\sum_{k=1}^{n}\left(\sum_{k_{1}+k_{2}=n-k}\left(\begin{array}{c}
n-1 \\
k_{1}, k_{2}, k-1
\end{array}\right) \lambda^{n-k} B_{k_{2}}^{(n)} B_{k_{1}}^{\left(n-k_{1}\right)}\right) x^{k} .
\end{aligned}
$$

By induction on $m$, we obtain the following result.

Theorem 3.1 For all $m, n \geq 1$,

$$
r_{n}^{(m)}(x)=\sum_{k=1}^{n}\left(\sum_{k_{1}+\cdots+k_{m}=n-k}\left(\begin{array}{c}
n-1 \\
k_{1}, \ldots, k_{m}, k-1
\end{array}\right) \lambda^{n-k} \prod_{j=1}^{m} B_{k_{j}}^{\left(n-\sum_{i=j+1}^{m} k_{i}\right)}\right) x^{k}
$$

Note that by combining the two expressions (see (4) and Theorem 3.1) for $r_{n}^{(m)}(x)$, we obtain the same result as obtained in [8], Theorem 4:

Corollary 3.2 For all $1 \leq k \leq n$ and $m \geq 1$,

$$
\begin{aligned}
& \sum_{\ell_{1}, \ldots, \ell_{m-1}=0}^{n} S_{1}\left(n, \ell_{1}\right) S_{1}\left(\ell_{1}, \ell_{2}\right) \cdots S_{1}\left(\ell_{m-1}, k\right) \\
= & \sum_{k_{1}+\cdots+k_{m}=n-k}\left(\begin{array}{c}
n-1 \\
k_{1}, \ldots, k_{m}, k-1
\end{array}\right) B_{k_{m}}^{(n)} B_{k_{m-1}}^{\left(n-k_{m}\right)} \cdots B_{k_{1}}^{\left(n-k_{m}-\cdots-k_{2}\right)} .
\end{aligned}
$$

Note that for $m=1$, the above corollary reduces to $S_{1}(n, k)=\left(\begin{array}{c}n-1 \\ k-1\end{array}\right) B_{n-k}^{(n)}$.

\subsection{Degenerate Bell polynomials}

Now, let us take the associated sequence $r_{n}(x)$ to $f(t)=\frac{(1+t)^{\lambda}-1}{\lambda}$. So $r_{n}(x)=\sum_{k=0}^{n} S_{2}(n$, $k \mid \lambda) x^{k} \sim(1, f(t))$ and $r_{n}^{(m)}(x)=\sum_{k=0}^{n} r_{n, k}^{(m)} x^{k} \sim\left(1, f^{m}(t)\right)$. Here, $S_{2}(n, k \mid \lambda)$ are the degenerate Stirling numbers of the second kind obtained by $\frac{1}{k !}\left((1+\lambda t)^{1 / \lambda}-1\right)^{k}=\sum_{n \geq k} S_{2}(n, k \mid \lambda) \frac{t^{n}}{n !}$ (see [9]). Indeed, as $\bar{f}(t)=(1+\lambda t)^{1 / \lambda}-1$, we get

$$
\begin{aligned}
\sum_{n \geq 0} r_{n}(x) \frac{t^{n}}{n !} & =e^{x\left((1+\lambda t)^{1 / \lambda}-1\right)}=\sum_{k \geq 0}\left((1+\lambda t)^{1 / \lambda}-1\right)^{k} \frac{x^{k}}{k !} \\
& =\sum_{n \geq 0}\left(\sum_{k=0}^{n} S_{2}(n, k \mid \lambda) x^{k}\right) \frac{t^{n}}{n !} .
\end{aligned}
$$

Thus, $r_{n}(x)=\sum_{k=0}^{n} S_{2}(n, k \mid \lambda) x^{k}$. As $\lambda \rightarrow 0, r_{n}(x)$ tends to the Bell polynomial $\operatorname{Bel}_{n}(x)=$ $\sum_{k=0}^{n} S_{2}(n, k) x^{k}$. Hence they may be called the degenerate Bell polynomials. From (3), we obtain

$$
r_{n, k}^{(m)}=\sum_{\ell_{1}, \ldots, \ell_{m-1}=0}^{n} S_{2}\left(n, \ell_{1} \mid \lambda\right) S_{2}\left(\ell_{1}, \ell_{2} \mid \lambda\right) \cdots S_{2}\left(\ell_{m-1}, k \mid \lambda\right) .
$$


On the other hand, from the transfer formula, we get, for $n \geq 1, r_{n}(x)=x\left(\lambda t /\left((1+t)^{\lambda}-\right.\right.$ 1) $)^{n} x^{-1} x^{n}$.

Recall that the Korobov polynomials $K_{n,(r)}(\lambda, x)$ of order $r$ (see [10]) are given by generating function $\left(\lambda t /\left((1+t)^{\lambda}-1\right)\right)^{r}(1+t)^{n}=\sum_{n \geq 0} K_{n,(r)}(\lambda, x) \frac{t^{n}}{n !}$. For $x=0, K_{n,(r)}(\lambda)=K_{n,(r)}(\lambda, 0)$ are called the Korobov numbers of order $r$. Note that $K_{n,(r)}(\lambda, x)$ should be distinguished from $K_{n}^{(r)}(\lambda, x)$, which denotes the $r$ th power under umbral composition of $K_{n}(\lambda, x)$. Thus,

$$
r_{n}(x)=x \sum_{k \geq 0} K_{k,(n)}(\lambda) \frac{t^{k}}{k !} x^{n-1}=\sum_{k=1}^{n}\left(\begin{array}{c}
n-1 \\
k-1
\end{array}\right) K_{n-k,(n)}(\lambda) x^{k} .
$$

To proceed further, we observe the following: for $0 \leq m \leq n-1$,

$$
\begin{aligned}
f(t)^{m} x^{-1} r_{n}(x) & =f(t)^{m}(t / f(t))^{n} x^{n-1}=(t / f(t))^{n-m} t^{m} x^{n-1} \\
& =(n-1)_{m}\left(\lambda t /\left((1+t)^{\lambda}-1\right)\right)^{n-m} x^{n-1-m} \\
& =(n-1)_{m} \sum_{\ell \geq 0} K_{\ell,(n-m)}(\lambda) \frac{t^{\ell}}{\ell !} x^{n-1-m} \\
& =\sum_{\ell=0}^{n-1-m} \frac{(n-1)_{\ell+m}}{\ell !} K_{\ell,(n-m)}(\lambda) x^{n-1-\ell-m} .
\end{aligned}
$$

Thus, by induction on $m$ and (3), we can state the following formula.

Theorem 3.3 For all $m, n \geq 1$,

$$
r_{n}^{(m)}(x)=\sum_{k=1}^{n}\left(\sum_{k_{1}+\cdots+k_{m}=n-k}\left(\begin{array}{c}
n-1 \\
k_{1}, \ldots, k_{m}, k-1
\end{array}\right) \prod_{j=1}^{m} K_{k_{j},\left(n-\sum_{i=j+1}^{m} k_{i}\right)}(\lambda)\right) x^{k} .
$$

Combining the two expressions for $r_{n}^{(m)}(x)$ (see (6) and Theorem 3.3), we obtain the following corollary.

Corollary 3.4 For all $1 \leq k \leq n$ and $m \geq 1$,

$$
\begin{aligned}
& \sum_{\ell_{1}, \ldots, \ell_{m-1}=0}^{n} S_{2}\left(n, \ell_{1} \mid \lambda\right) S_{2}\left(\ell_{1}, \ell_{2} \mid \lambda\right) \cdots S_{2}\left(\ell_{m-1}, k \mid \lambda\right) \\
& =\sum_{k_{1}+\cdots+k_{m}=n-k}\left(\begin{array}{c}
n-1 \\
k_{1}, \ldots, k_{m}, k-1
\end{array}\right) \prod_{j=1}^{m} K_{k_{j},\left(n-\sum_{i=j+1}^{m} k_{i}\right)}(\lambda) .
\end{aligned}
$$

Note that the above corollary with $m=1$ shows that $S_{2}\left(n, \ell_{1} \mid \lambda\right)=\left(\begin{array}{c}n-1 \\ k-1\end{array}\right) K_{n-k,(n)}(\lambda)$.

\subsection{Degenerate falling factorial polynomials}

As for third example, let us consider the associated sequence $r_{n}(x)$ to $f(t)=(1+\lambda t)^{1 / \lambda}-$ 1. So, $r_{n}(x)=\sum_{k=0}^{n} S_{1}(n, k \mid \lambda) x^{k} \sim(1, f(t))$ and $r_{n}^{(m)}(x)=\sum_{k=0}^{n} r_{n, k}^{(m)} x^{k} \sim\left(1, f^{m}(t)\right)$. Here, $S_{1}(n, k \mid \lambda)$ are the degenerate Stirling numbers of the first kind (see $\left.[4,9]\right)$ given by $\frac{1}{k !}((1+$ $\left.t)^{\lambda}-1\right)^{k} / \lambda^{k}=\sum_{n \geq k} S_{1}(n, k \mid \lambda) \frac{t^{n}}{n !} \cdot r_{n}(x)$ may be called the degenerate falling factorial polynomials, since, as $\lambda \rightarrow 0, r_{n}(x)$ tends to the falling factorial polynomial $(x)_{n}=\sum_{k=0}^{n} S_{1}(n, k) x^{k}$. 
From (3), we obtain

$$
r_{n, k}^{(m)}=\sum_{\ell_{1}, \ldots, \ell_{m-1}=0}^{n} S_{1}\left(n, \ell_{1} \mid \lambda\right) S_{1}\left(\ell_{1}, \ell_{2} \mid \lambda\right) \cdots S_{1}\left(\ell_{m-1}, k \mid \lambda\right) .
$$

We recall that the degenerate Bernoulli polynomials $\beta_{n,(r)}(\lambda, x)$ of order $r$ are defined by the generating function

$$
t^{r} /\left((1+\lambda t)^{1 / \lambda}-1\right)^{r}(1+\lambda t)^{x / \lambda}=\sum_{n \geq 0} \beta_{n,(r)}(\lambda, x) \frac{t^{n}}{n !} .
$$

For $x=0, \beta_{n,(r)}(\lambda)=\beta_{n,(r)}(\lambda, 0)$ are called the degenerate Bernoulli numbers of order $r$. Here, $\beta_{n,(r)}(\lambda, x)$ should not be confused with $\beta_{n}^{(r)}(\lambda, x)$, which denotes the $r$ th power under umbral composition of $\beta_{n}(\lambda, x)$. So, by these definitions, for $n \geq 1$, we have

$$
\begin{aligned}
r_{n}(x) & =x \frac{t^{n}}{\left((1+\lambda t)^{1 / \lambda}-1\right)^{n}} x^{n-1}=x \sum_{k \geq 0} \beta_{k,(n)}(\lambda) \frac{t^{k}}{k !} x^{n-1} \\
& =\sum_{k=1}^{n}\left(\begin{array}{l}
n-1 \\
k-1
\end{array}\right) \beta_{n-k,(n)}(\lambda) x^{k} .
\end{aligned}
$$

Thus, for $0 \leq m \leq n-1$, we have

$$
\begin{aligned}
f(t)^{m} x^{-1} r_{n}(x) & =(t / f(t))^{n-m} t^{m} x^{n-1} \\
& =(n-1)_{m} \frac{t^{n-m}}{\left((1+\lambda t)^{1 / \lambda}-1\right)^{n-m}} x^{n-1-m} \\
& =\sum_{\ell=0}^{n-1-m} \frac{(n-1)_{\ell+m}}{\ell !} \beta_{\ell,(n-m)}(\lambda) x^{n-1-\ell-m} .
\end{aligned}
$$

By using similar arguments to (5), we obtain

$$
\begin{aligned}
r_{n}^{(2)}(x) & =x\left(f(t) / f^{2}(t)\right)^{n} x^{-1} r_{n}(x) \\
& =\sum_{k=1}^{n}\left(\sum_{k_{+} k_{2}=n-k}\left(\begin{array}{c}
n-1 \\
k_{1}, k_{2}, k-1
\end{array}\right) \beta_{k_{2},(n)}(\lambda) \beta_{k_{1},\left(n-k_{2}\right)}(\lambda)\right) x^{k} .
\end{aligned}
$$

Hence, by induction on $m$, we derive the following result.

Theorem 3.5 For all $m, n \geq 1$,

$$
r_{n}^{(m)}(x)=\sum_{k=1}^{n}\left(\sum_{k_{1}+\cdots+k_{m}=n-k}\left(\begin{array}{c}
n-1 \\
k_{1}, \ldots, k_{m}, k-1
\end{array}\right) \prod_{j=1}^{m} \beta_{k_{j},\left(n-\sum_{i=j+1}^{m} k_{i}\right)}(\lambda)\right) x^{k}
$$

Combining the two expressions for $r_{n}^{(m)}(x)$ (see (7) and Theorem 3.5), we obtain the following corollary. 
Corollary 3.6 For all $1 \leq k \leq n$ and $m \geq 1$,

$$
\begin{aligned}
& \sum_{\ell_{1}, \ldots, \ell_{m-1}=0}^{n} S_{1}\left(n, \ell_{1} \mid \lambda\right) S_{1}\left(\ell_{1}, \ell_{2} \mid \lambda\right) \cdots S_{1}\left(\ell_{m-1}, k \mid \lambda\right) \\
& =\sum_{k_{1}+\cdots+k_{m}=n-k}\left(\begin{array}{c}
n-1 \\
k_{1}, \ldots, k_{m}, k-1
\end{array}\right) \prod_{j=1}^{m} \beta_{k_{j},\left(n-\sum_{i=j+1}^{m} k_{i}\right)}(\lambda) .
\end{aligned}
$$

Note that the above corollary with $m=1$ shows that $S_{1}(n, k \mid \lambda)=\left(\begin{array}{c}n-1 \\ k-1\end{array}\right) \beta_{n-k,(n)}(\lambda)$.

\subsection{Generalized Bell polynomials}

One more example is Bell polynomials, also called the exponential polynomials, which are given by the generating function $e^{x\left(e^{t}-1\right)}=\sum_{n \geq 0} B e l_{n}(x) \frac{t^{n}}{n !}$ (see [4]). Let $r_{n}(x)=$ $\lambda^{n} \operatorname{Bel}_{n}(x / \lambda)=\sum_{k=0}^{n} \lambda^{n-k} S_{n}(n, k) x^{k} \sim(1, f(t))$ with $f(t)=\frac{1}{\lambda} \log (1+\lambda t)$. Thus, $\bar{f}(t)=\frac{e^{\lambda t}-1}{\lambda}$, which implies

$$
\begin{aligned}
\sum_{n \geq 0} r_{n}(x) \frac{t^{n}}{n !} & =e^{\frac{x}{\lambda}\left(e^{\lambda t}-1\right)}=\sum_{n \geq 0} \lambda^{n} \operatorname{Bel}_{n}(x / \lambda) \frac{t^{n}}{n !} \\
& =\sum_{n \geq 0}\left(\sum_{k=0}^{n} \lambda^{n-k} S_{2}(n, k) x^{k}\right) \frac{t^{n}}{n !} .
\end{aligned}
$$

Thus, from (3), we get

$$
r_{n, k}^{(m)}=\lambda^{n-k} \sum_{\ell_{1}, \ldots, \ell_{m-1}=0}^{n} S_{2}\left(n, \ell_{1}\right) S_{2}\left(\ell_{1}, \ell_{2}\right) \cdots S_{2}\left(\ell_{m-1}, k\right) .
$$

To proceed we recall that the Bernoulli polynomials of the second kind $b_{n,(r)}(x)$ of order $r$ are given by the generating function

$$
(t / \log (1+t))^{r}(1+t)^{x}=\sum_{n \geq 0} b_{n,(r)}(x) \frac{t^{n}}{n !}
$$

(see [4]). For $x=0, b_{n,(r)}=b_{n,(r)}(0)$ are the Bernoulli numbers of the second kind of order $r$. Here, $b_{n,(r)}(x)$ should be distinguished from $b_{n}^{(r)}(x)$, which denotes the $r$ th power under umbral composition of $b_{n}(x)$. So, for $n \geq 1$,

$$
\begin{aligned}
r_{n}(x) & =x\left(\frac{\lambda t}{\log (1+\lambda t)}\right)^{n} x^{n-1}=x \sum_{k \geq 0} b_{k,(n)} \frac{\lambda^{k} t^{k}}{k !} x^{n-1} \\
& =\sum_{k=1}^{n}\left(\begin{array}{c}
n-1 \\
k-1
\end{array}\right) \lambda^{n-k} b_{n-k,(n)} x^{k} .
\end{aligned}
$$

For $0 \leq m \leq n-1$, we observe that

$$
f(t)^{m} x^{-1} r_{n}(x)=(t / f(t))^{n-m} t^{m} x^{n-1}=\sum_{\ell=0}^{n-1-m} \frac{(n-1)_{\ell+m}}{\ell !} \lambda^{\ell} b_{\ell,(n-m)} x^{n-1-\ell-m} .
$$

Thus, by induction on $m$ (similar to Theorem 3.1), one can obtain the following formula. 
Theorem 3.7 For all $m, n \geq 1$,

$$
r_{n}^{(m)}(x)=\sum_{k=1}^{n}\left(\sum_{k_{1}+\cdots+k_{m}=n-k} \lambda^{n-k}\left(\begin{array}{c}
n-1 \\
k_{1}, \ldots, k_{m}, k-1
\end{array}\right) \prod_{j=1}^{m} b_{k_{j},\left(n-\sum_{i=j+1}^{m} k_{i}\right)}\right) x^{k} .
$$

Combining the two expressions for $r_{n}^{(m)}(x)$ (see (8) and Theorem 3.7), we obtain the following corollary.

Corollary 3.8 For all $1 \leq k \leq n$ and $m \geq 1$,

$$
\begin{aligned}
& \sum_{\ell_{1}, \ldots, \ell_{m-1}=0}^{n} S_{2}\left(n, \ell_{1}\right) S_{2}\left(\ell_{1}, \ell_{2}\right) \cdots S_{2}\left(\ell_{m-1}, k\right) \\
& =\sum_{k_{1}+\cdots+k_{m}=n-k}\left(\begin{array}{c}
n-1 \\
k_{1}, \ldots, k_{m}, k-1
\end{array}\right) \prod_{j=1}^{m} b_{k_{j},\left(n-\sum_{i=j+1}^{m} k_{i}\right)} .
\end{aligned}
$$

Note that the above corollary with $m=1$ shows that $S_{2}(n, k)=\left(\begin{array}{c}n-1 \\ k-1\end{array}\right) b_{n-k,(n)}$.

\section{Degenerations of Sheffer sequences}

Let $s_{n}(x) \sim(g(t), f(t))$ and $r_{n}(x) \sim(1, \ell(t))$. Then $g(\bar{f}(t))^{-1} e^{x \bar{f}(t)}=\sum_{n \geq 0} s_{n}(x) \frac{t^{n}}{n !}$. In view of Theorem 2.1 and (1), we have

$$
g\left(\bar{f}\left(\bar{\ell}^{m}(t)\right)\right)^{-1} e^{x \bar{f}\left(\bar{\ell}^{m}(t)\right)}=\sum_{n \geq 0} r_{n}^{(m)} \circ s_{n}(x) \frac{t^{n}}{n !}
$$

In other words, the generating function for $r_{n}^{(m)} \circ s_{n}(x)$ is obtained from that of $s_{n}(x)$ by replacing $t$ by $\bar{\ell}(t)$ exactly $m$ times. In particular, for $\ell(t)=\frac{1}{\lambda}\left(e^{\lambda t}-1\right)\left(\right.$ resp. $\ell(t)=\frac{1}{\lambda} \log (1+$ $\lambda t)), r_{n}^{(m)} \circ s_{n}(x)$ will be called the $m$ th degeneration of $s_{n}(x)$ by $\bar{\ell}(t)=\frac{1}{\lambda} \log (1+\lambda t)$ (resp. $\left.\ell(t)=\frac{1}{\lambda}\left(e^{\lambda t}-1\right)\right)$.

The research in this section was motivated by the following example (see [8]) $b_{n}(x) \sim$ $\left(t /\left(e^{t}-1\right), e^{t}-1\right)=(g(t), f(t))$ and $r_{n}(x)=(x \mid \lambda)_{n} \sim\left(1,\left(e^{\lambda t}-1\right) / \lambda\right)=(1, \ell(t))$. Note here that $b_{n}(x)$ is the Bernoulli polynomial of the second kind whose generating function is given by

$$
\frac{t}{\log (1+t)}(1+t)^{x}=\sum_{n \geq 0} b_{n}(x) \frac{t^{n}}{n !}
$$

From this consideration, we see that the generating function of $r_{n}^{(m)} \circ b_{n}(x)$, the $m$ th degeneration of $b_{n}(x)$ by $\bar{\ell}(t)=\frac{1}{\lambda} \log (1+\lambda t)$, is obtained from $b_{n}(x)$ in (9) by replacing $t$ by $\frac{1}{\lambda} \log (1+\lambda t)$ exactly $m$ times. The polynomial $r_{n}^{(m)} \circ b_{n}(x)$ is denoted by $c_{n, \lambda}^{(m)}(x)$ in [8] and is given by

$$
c_{n, \lambda}^{(m)}(x)=\sum_{k=0}^{n} \lambda^{n-k}\left(\sum_{\ell_{1}, \ldots, \ell_{m-1}=0}^{n} S_{1}\left(n, \ell_{1}\right) S_{1}\left(\ell_{1}, \ell_{2}\right) \cdots S_{1}\left(\ell_{m-1}, k\right)\right) b_{k}(x) .
$$

This agrees with the result in [8]. 
In general, the $m$ th degeneration of $s_{n}(x) \sim(g(t), f(t))$ by $\bar{\ell}(t)=\frac{1}{\lambda} \log (1+\lambda t)$ is given by

$$
r_{n}^{(m)} \circ s_{n}(x)=\sum_{k=0}^{n} \lambda^{n-k}\left(\sum_{\ell_{1}, \ldots, \ell_{m-1}=0}^{n} S_{1}\left(n, \ell_{1}\right) S_{1}\left(\ell_{1}, \ell_{2}\right) \cdots S_{1}\left(\ell_{m-1}, k\right)\right) s_{k}(x)
$$

where $r_{n}(x) \sim\left(1,\left(e^{\lambda t}-1\right) / \lambda\right)=(1, \ell(t))$.

Also, the $m$ th degeneration of $s_{n}(x) \sim(g(t), f(t))$ by $\bar{\ell}(t)=\frac{1}{\lambda}\left(e^{\lambda t}-1\right)$ is given by

$$
r_{n}^{(m)} \circ s_{n}(x)=\sum_{k=0}^{n} \lambda^{n-k}\left(\sum_{\ell_{1}, \ldots, \ell_{m-1}=0}^{n} S_{2}\left(n, \ell_{1}\right) S_{2}\left(\ell_{1}, \ell_{2}\right) \cdots S_{2}\left(\ell_{m-1}, k\right)\right) s_{k}(x),
$$

where $r_{n}(x) \sim(1, \log (1+\lambda t) / \lambda)=(1, \ell(t))$. On the other hand,

$$
r_{n}^{(m)} \circ s_{n}(x)=\sum_{k=0}^{n}\left(\sum_{\ell_{1}, \ldots, \ell_{m-1}=0}^{n} S_{2}\left(n, \ell_{1} \mid \lambda\right) S_{2}\left(\ell_{1}, \ell_{2} \mid \lambda\right) \cdots S_{2}\left(\ell_{m-1}, k \mid \lambda\right)\right) s_{k}(x)
$$

are polynomials whose generating function is obtained from that of $s_{n}(x) \sim(g(t), f(t))$ by replacing $t$ by $\bar{\ell}(t)=(1+\lambda t)^{1 / \lambda}-1$ exactly $m$ times (here $\left.r_{n}(x) \sim(1, \ell(t))=\left(1,\left((1+t)^{\lambda}-1\right) / \lambda\right)\right)$.

In addition,

$$
r_{n}^{(m)} \circ s_{n}(x)=\sum_{k=0}^{n}\left(\sum_{\ell_{1}, \ldots, \ell_{m-1}=0}^{n} S_{1}\left(n, \ell_{1} \mid \lambda\right) S_{1}\left(\ell_{1}, \ell_{2} \mid \lambda\right) \cdots S_{1}\left(\ell_{m-1}, k \mid \lambda\right)\right) s_{k}(x)
$$

are the polynomials whose generating function is obtained from that of $s_{n}(x) \sim(g(t), f(t))$ by replacing $t$ by $\bar{\ell}(t)=\left((1+t)^{\lambda}-1\right) / \lambda$ exactly $m$ times $\left(\right.$ here $r_{n}(x) \sim(1, \ell(t))=\left(1,(1+\lambda t)^{1 / \lambda}-\right.$ 1)).

\section{Competing interests}

The authors declare that they have no competing interests.

\section{Authors' contributions}

All authors contributed equally to this work. All authors read and approved the final manuscript.

\section{Author details}

'Department of Mathematics, Sogang University, Seoul, 121-742, South Korea. ²Department of Mathematics, College of Science, Tianjin Polytechnic University, Tianjin, 300387, China. ${ }^{3}$ Department of Mathematics, Kwangwoon University, Seoul, 139-701, South Korea. ${ }^{4}$ Department of Mathematics, University of Haifa, Haifa, 3498838, Israel.

\section{Acknowledgements}

The authors express their sincere gratitude to the two anonymous referees for the careful reading of the original manuscript and useful comments that helped to improve the presentation of the paper.

Received: 13 October 2015 Accepted: 28 February 2016 Published online: 02 March 2016

\section{References}

1. Ruiz, JA: Riordan arrays from an umbral symbolic viewpoint. Bol. Soc. Port. Mat. 2012, 5-8 (2012)

2. Roman, S: The Umbral Calculus. Dover, New York (2005)

3. Kim, DS, Kim, T, Ryoo, CS: Sheffer sequences for the powers of Sheffer pairs under umbral composition. Adv. Stud. Contemp. Math. 23(2), 275-285 (2013)

4. Kim, DS, Kim, T: Higher-order degenerate Bernoulli polynomials. Preprint

5. Araci, S, Acikgoz, M: A note on the Frobenius-Euler numbers and polynomials associated with Bernstein polynomials. Adv. Stud. Contemp. Math. 22(3), 399-406 (2012)

6. He, Y, Wang, SJ: New formulae of products of the Frobenius-Euler polynomials. Adv. Differ. Equ. 2014,261 (2014)

7. Simsek, Y: Generating functions for q-Apostol type Frobenius-Euler numbers and polynomials. Axioms 1, 395-403 (2012) 
8. Jeong, J, Rim, SH, Kim, BM: On finite times degenerate Cauchy numbers and polynomials. Adv. Differ. Equ. 2015,321 (2015)

9. Carlitz, L: Degenerate Stirling, Bernoulli and Eulerian numbers. Util. Math. 15, 51-88 (1979)

10. Dolgy, DV, Kim, DS, Kim, T: On the Korobov polynomials of the first kind. Preprint

Submit your manuscript to a SpringerOpen ${ }^{\circ}$ journal and benefit from:

- Convenient online submission

Rigorous peer review

- Immediate publication on acceptance

- Open access: articles freely available online

- High visibility within the field

Retaining the copyright to your article

Submit your next manuscript at $>$ springeropen.com 